\title{
Adhesion of Stenotrophomonas maltophilia to mouse tracheal mucus is mediated through flagella
}

\author{
Correspondence \\ Sanjay Chhibber \\ chhibbersanjay@ymail.com
}

Received 21 September 2010

Accepted 14 March 2011

\author{
Ayaid Khadem Zgairt and Sanjay Chhibber
}

Department of Microbiology, BMS Block, Panjab University, Chandigarh 160014, India

\section{INTRODUCTION}

Bacterial adhesion is an essential step in the pathogenesis of infections of mucosal and prosthetic surfaces ( $\mathrm{Di}$ Bonaventura et al., 2004). However, the molecular and physical interactions that govern bacterial adhesion to these surfaces require an understanding of both specific and non-specific interactions (Katsikogianni \& Missirlis, 2004; Oztuna et al., 2006). Stenotrophomonas maltophilia strains of both clinical and environmental origin have been reported to adhere to and form biofilm on abiotic surfaces such as glass, Teflon, polystyrene and stainless steel (Jucker et al., 1996; Baker \& Leff, 2005). However, information regarding their ability to adhere to mucus is lacking in the literature. Adhesion of bacteria to mucus has been considered an important step in the pathogenesis of respiratory tract infections (Sajjan et al., 1992). As S. maltophilia is an organism frequently associated with infections of the respiratory tract, it is imperative to study its ability to adhere to mucus.

Mucus that coats the surfaces of the gastrointestinal, respiratory and reproductive tracts provides an outermost

tPresent address: Department of Biology - Microbiology, Baghdad University, Baghdad, Iraq. barrier against pathogens. It contains glycoproteins that are composed of a peptide backbone linked to carbohydrates, which act as a receptor for bacterial adhesins. This interaction promotes adherence, as has been shown for Pseudomonas aeruginosa (Arora et al., 1998).

In the present study, an attempt was made to study the ability of five clinical isolates of $S$. maltophilia to adhere to mouse tracheal mucus and to understand the underlying mechanism of adhesion.

\section{METHODS}

Clinical isolates. Five clinical isolates of $S$. maltophilia $(\mathrm{Sm} 1, \mathrm{Sm} 2$, Sm3, Sm6, Sm7) were used in this study. These isolates were obtained from the Department of Medical Microbiology, Post Graduate Institute of Medical Education and Research, Chandigarh, India. The bacteria were preserved by lyophilization, routinely cultured at $37^{\circ} \mathrm{C}$ on Luria-Bertani agar plates and subcultured every week.

Flagellin and anti-flagellin preparation. Flagellin was isolated from S. maltophilia isolate $\mathrm{Sm} 2$ as described previously (Chhibber \& Zgair, 2009). Briefly, S. maltophilia Sm2 was grown overnight and pelleted by centrifugation. The pellet was suspended in $0.01 \mathrm{M}$ potassium phosphate buffer ( $\mathrm{pH}$ 7.0) and sheared for $1 \mathrm{~min}$ in a commercial blender. The sheared suspension was centrifuged for $30 \mathrm{~min}$ at $5000 \mathrm{~g}$ and then for 
$15 \mathrm{~min}$ at $16000 \mathrm{~g}\left(4^{\circ} \mathrm{C}\right)$. The supernatant was centrifuged at $100000 \mathrm{~g}$ for $3 \mathrm{~h}\left(4{ }^{\circ} \mathrm{C}\right)$. The pellet was collected and kept at $-80{ }^{\circ} \mathrm{C}$. Anti-flagellin antiserum was prepared by immunizing a rabbit with pure flagellin. Complement was inactivated by incubating the sera for $30 \mathrm{~min}$ at $56{ }^{\circ} \mathrm{C}$.

Transmission electron microscopy. The presence of flagella was detected by transmission electron microscopy. Flagella were negatively stained for 2 min with $1 \%$ phosphotungstic acid ( $\mathrm{pH} 7.4$ ) on carbon/Formvar copper grids (Electron Microscopy Sciences) as described previously (Girón, 1995).

Animals. $\mathrm{BALB} / \mathrm{c}$ mice, $6-8$ weeks old, weighing $20-25 \mathrm{~g}$ were obtained from the central animal house of Panjab University, Chandigarh, India. The animals were kept in clean polypropylene cages and fed on a standard antibiotic-free diet (JBD Agencies). The study was conducted following approval from the animal ethics committee of Panjab University, Chandigarh. India.

Crude mucus preparations. Twenty microlitres of sterile PBS $(0.2 \mathrm{M}, \mathrm{pH} 7.2)$ was passed repeatedly through the tracheal lumen of the mice to wash out tracheal mucus. The slightly turbid mucus suspension was pooled, centrifuged at $10000 \mathrm{~g}$ for $10 \mathrm{~min}$ and filtered through a $0.2 \mu \mathrm{m}$ Millipore filter to remove cells and debris.

Assay of bacterial adherence to tracheal mucus. The bacterial isolates were grown in tryptic soy broth (HiMedia Laboratories) overnight at $37^{\circ} \mathrm{C}$ and the bacterial count was adjusted to $10^{7}$ c.f.u. $\mathrm{ml}^{-1}$. Microtitre plates (Nunc) were coated with crude mucus at a concentration of $100 \mu \mathrm{g} \mathrm{ml}^{-1}$ (Vishwanath \& Ramphal, 1984). Bacteria were added to each well and the plates were incubated at $37^{\circ} \mathrm{C}$ for $1 \mathrm{~h}$ to check the ability of the different clinical isolates to adhere to mucus. To study the kinetics of adhesion, samples were withdrawn at different time intervals $(0.5,1,2,4,8$ and $24 \mathrm{~h})$ after washing the wells five times with $0.2 \mathrm{M}$ PBS ( $\mathrm{pH}$ 7.2). The adherent bacteria were removed with $0.05 \%$ Triton $\mathrm{X}-100$ and enumerated by plating different dilutions on Luria-Bertani plates. All experiments were conducted in triplicate.

Adhesion inhibition assay. The bacterial isolate showing maximum adherence $(\mathrm{Sm} 2)$ was pre-treated for $30 \mathrm{~min}$ with different dilutions of anti-flagellin antibody $(1: 40,1: 80,1: 160,1: 320$ and $1: 640)$ and tested for adherence to mouse tracheal mucus as described above. In another experiment, wells coated with mouse tracheal mucus were pre-treated first with pure flagellin using different concentrations (10, 20, 40 and $80 \mu \mathrm{g} \mathrm{ml}^{-1}$ ) for $30 \mathrm{~min}$ to block the receptors and the adherence of isolate $\mathrm{Sm} 2$ was then evaluated by following the protocol described above.

Adhesion of flagella-sheared bacteria. After overnight growth, the bacteria were centrifuged, washed and deflagellated using a commercial blender for $1 \mathrm{~min}$. The ability of the sheared bacteria to adhere to mouse tracheal mucus was then determined. The results were compared with those obtained using non-sheared bacteria as a control.

Role of S. maltophilia flagellin in attachment to mouse tracheal mucus as evaluated by enzyme immunoassay. The method of Cogan et al. (2004) was followed with modifications. Briefly, four rows of a microtitre plate were used. Rows 1-3 were coated with mouse tracheal mucus as described above, whereas row 4 was left uncoated. Each row was washed four times with $0.2 \mathrm{M}$ PBS $(\mathrm{pH} 7.2)$ containing $0.05 \%$ Tween 20. S. maltophilia flagellin $(100 \mu \mathrm{l}$ of $20 \mu \mathrm{g} \mathrm{ml}^{-1}$ ) was added to each well of rows 1 and 2 and incubated for $1 \mathrm{~h}$ at $37^{\circ} \mathrm{C}$. No flagellin was added to rows 3 and 4 . After washing with PBS, the wells were blocked with $200 \mu \mathrm{l}$ PBS containing $3 \%$ dried milk and incubated at $37^{\circ} \mathrm{C}$ for $1 \mathrm{~h}$. Rabbit $S$. maltophilia anti-flagellin antibody (100 $\mu \mathrm{l}$ of $1: 320$ dilution) suspended in PBS containing $0.1 \%$ dried milk was added to each well of rows 1,3 and 4 , whilst normal rabbit serum was added to the wells of row 2 (control) and the plate was incubated at $37{ }^{\circ} \mathrm{C}$ for another $1 \mathrm{~h}$. Goat anti-rabbit horseradish peroxidase-conjugated antibody (1:1000; Sigma) was added to the wells and incubated for $1 \mathrm{~h}$, followed by the addition of tetramethylbenzidine substrate (Sigma). The reaction was stopped with $2 \mathrm{M}$ sulphuric acid (Fluka) and the $A_{450}$ was read. Thus, in this experiment, row 1 was the test row, row 2 was control 1 (CN1: mucus + flagellin + normal rabbit serum), row 3 was control 2 (CN2: mucus + anti-flagellin antibody only) and row 4 was control 3 (CN3: anti-flagellin antibody only).

In another experiment, flagellin pre-treated with different dilutions of anti-flagellin antibody was applied to mucus-coated wells instead of flagellin. The control well consisted of mucus, flagellin and antiflagellin antibody.

Statistical analysis. All values were calculated as the means \pm SEM. Differences between tests and controls were analysed using Student's $t$-test and the correlation coefficient using Origin version 8.0 software. A value of $P<0.05$ was considered statistically significant.

\section{RESULTS}

\section{Adhesion of clinical isolates to mouse tracheal mucus}

The results presented in Fig. 1 show that all five clinical isolates tested in this study were able to adhere to mouse tracheal mucus. Maximum adherence was observed with S. maltophilia isolate $\mathrm{Sm} 2$ followed by $\mathrm{Sm} 3$, and was least with $\mathrm{Sm} 1$.

\section{Confirmation of the presence of flagella}

Transmission electron microscopic examination of the bacteria confirmed the expression of flagella. Some of the bacteria expressed only one polar flagellum, whilst others expressed more than one (Fig. 2a). Pure flagellar preparation contained numerous such flagella without any bacterial contamination (Fig. 2b).

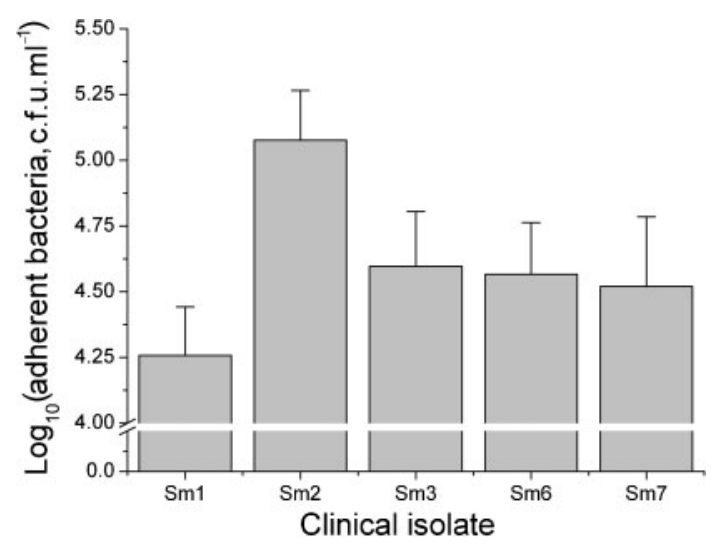

Fig. 1. Ability of different clinical isolates of $S$. maltophilia to adhere to mouse tracheal mucus. Maximum adherence was observed with isolate Sm2 and minimum with Sm1. 


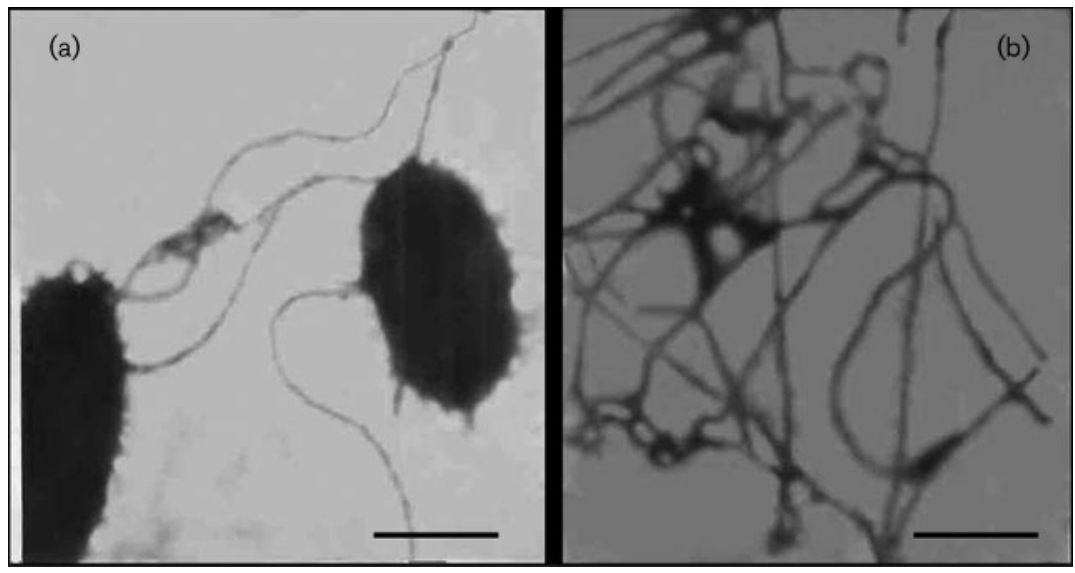

Fig. 2. Transmission electron micrographs showing the polar arrangement of flagella on S. maltophilia cells (a) and the purity of the flagellar preparation (b). The cells and polar flagellar preparation were negatively stained. Bars, $0.5 \mu \mathrm{m}$ (a) and $0.25 \mu \mathrm{m}$ (b).

\section{Kinetics of S. maltophilia adhesion to mouse tracheal mucus}

The Sm2 isolate of S. maltophilia was used to study the kinetics of adhesion, as maximum adhesion to mouse tracheal mucus was seen with this isolate. The results presented in Fig. 3 showed that bacterial adhesion started after $30 \mathrm{~min}$ of incubation, but significant adhesion to mouse tracheal mucus was detected after $1 \mathrm{~h}(P<0.05)$. The increase in bacterial numbers continued thereafter and the maximum count was obtained at $24 \mathrm{~h}$.

\section{Effect of S. maltophilia flagellin on bacterial adhesion to mouse tracheal mucus}

To study the involvement of $S$. maltophilia flagellin as a bacterial adhesin to mouse tracheal mucus, S. maltophilia Sm2 was pre-treated with anti-flagellin antibody for

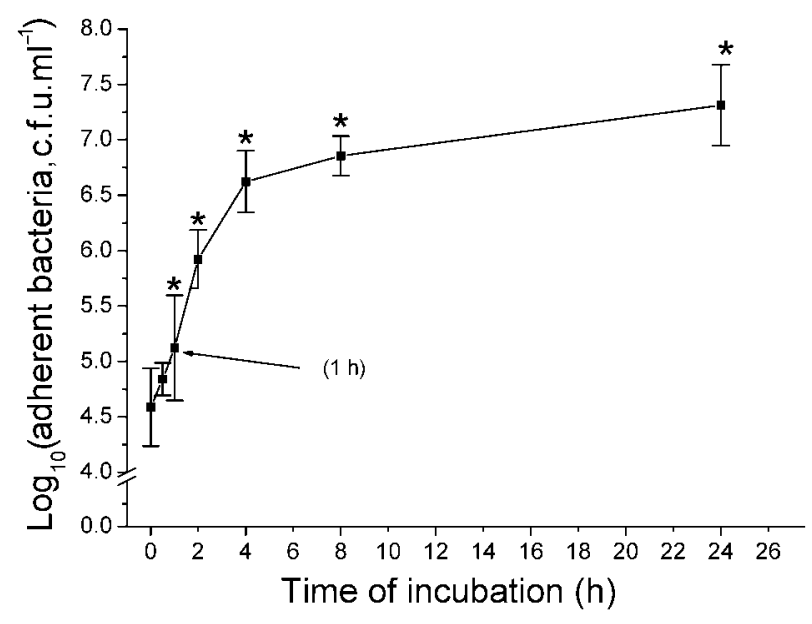

Fig. 3. Kinetics of adhesion of S. maltophilia isolate $S m 2$ to mouse tracheal mucus. Significant adhesion of bacteria to the mucus surface was detected after $1 \mathrm{~h}$ of incubation. Asterisks indicate a significant difference compared with adhesion at $0 \mathrm{~h}(P<0.05)$.
30 min before the binding assay. As shown in Fig. 4, pretreatment of the bacteria with antibody resulted in decreased adherence of $S$. maltophilia to mouse tracheal mucus. A significant reduction in adhesion was seen compared with the control at all concentrations tested $(P<0.05)$. A negative relationship was detected between anti-flagellin antibody concentration and bacterial adhesion to mouse tracheal mucus, measured in c.f.u. $\mathrm{ml}^{-1}$ (correlation coefficient -0.97 ).

A decrease in adhesion of S. maltophilia Sm2 to mouse tracheal mucus was observed after pre-treatment of the mucus for $30 \mathrm{~min}$ with pure flagellin compared with untreated mucus $(P<0.05)$ (Fig. 5). This effect was

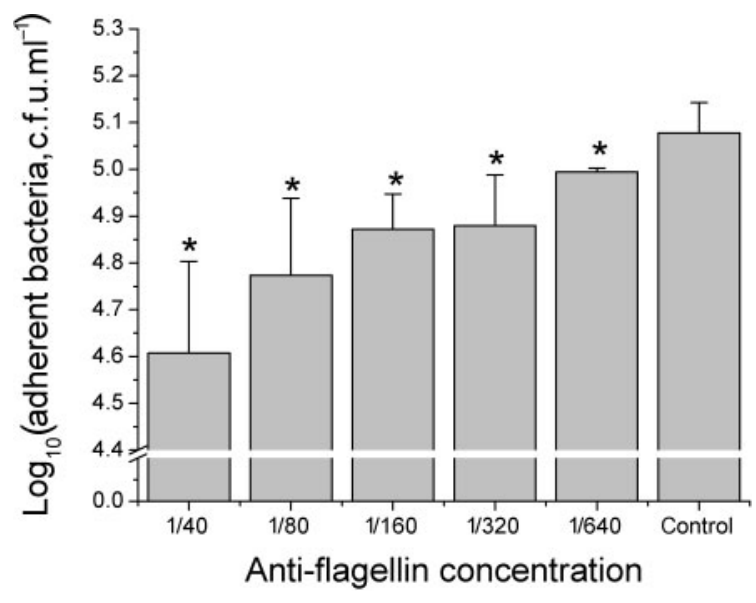

Fig. 4. Number of S. maltophilia $\left(\log _{10}\right.$ c.f.u. $\mathrm{ml}^{-1}$ ) adhering to mouse tracheal mucus following interaction of pre-treatment of the bacteria with different concentrations of anti-flagellin antibody. Interaction with the antibody significantly reduced the bacterial adherence to mouse tracheal mucus $(P<0.05)$. A negative relationship was detected between the anti-flagellin antibody concentration and bacterial adherence (correlation coefficient $-0.97)$. Asterisks indicate a significant difference compared with the control (no antibody) $(P<0.05)$. 


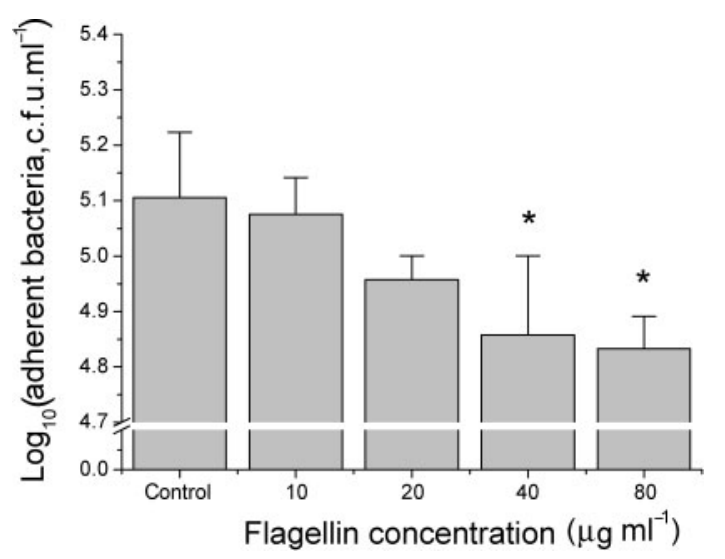

Fig. 5. Number of $S$. maltophilia $\left(\log _{10}\right.$ c.f.u. $\left.\mathrm{ml}^{-1}\right)$ adhering to mouse tracheal mucus following pre-treatment of the mucus with different concentrations of pure flagellin. Pre-treatment of mouse tracheal mucus with flagellin significantly reduced the bacterial adherence at concentrations of 40 and $80 \mu \mathrm{g} \mathrm{ml}^{-1}(P<0.05)$. A negative relationship was detected between flagellin concentration and bacterial adherence (correlation coefficient -0.85). Asterisks indicate a significant difference compared with the control (no flagellin) $(P<0.05)$.

significant at higher concentrations of flagellin (40 and $80 \mu \mathrm{g} \mathrm{ml}^{-1}$ ) but not at lower concentrations (10 and $20 \mu \mathrm{g}$ $\left.\mathrm{ml}^{-1}\right)$. A negative relationship was found between flagellin concentration and $S$. maltophilia adhesion to mouse tracheal mucus measured in c.f.u. $\mathrm{ml}^{-1}$ (correlation coefficient -0.85). On the basis of these results, we concluded that $S$. maltophilia flagellin is an adhesin for mouse tracheal mucus and that receptors for $S$. maltophilia flagellin are present on mouse tracheal mucus.

\section{Adhesion of flagella-sheared bacteria}

The role of flagella in the adhesion of S. maltophilia to mouse tracheal mucus was further checked by removing the bacterial flagella in a commercial blender. The results showed a significant decrease in bacterial adhesion of sheared bacteria compared with the adhesion seen with non-sheared bacteria $(P<0.005)$. The $\log _{10}$ value of the mechanically deflagellated bacteria was $4.28 \pm 0.27$ compared with $5.07 \pm 0.14$ c.f.u. $\mathrm{ml}^{-1}$ obtained with flagellated bacteria (control) on interaction with mucus.

\section{Attachment of S. maltophilia flagellin to mouse tracheal mucus}

An enzyme immunoassay was carried out to confirm the ability of $S$. maltophilia flagellin to bind to mucus. The results showed that flagellin was able to adhere to mucus directly (Fig. 6a). However, no adherence was observed in the controls that lacked either flagellin or anti-flagellin or both. The results confirmed that receptors for flagellin are present on mouse tracheal mucus. The results in Fig. 6(b) showed that attachment of S. maltophilia flagellin declined following pre-treatment with different dilutions of antiflagellin antibody. These results confirmed the presence of adhesins on S. maltophilia flagellin that were blocked following treatment with the antibody.

\section{DISCUSSION}

The results of this study clearly depict the ability of clinical isolates of $S$. maltophilia to adhere to mucus present on mouse respiratory epithelium. Mucus secreted by specialized epithelial cells in the respiratory tract forms a protective covering, reducing the ability of bacteria to adhere to epithelial cells and thus checking the initiation of
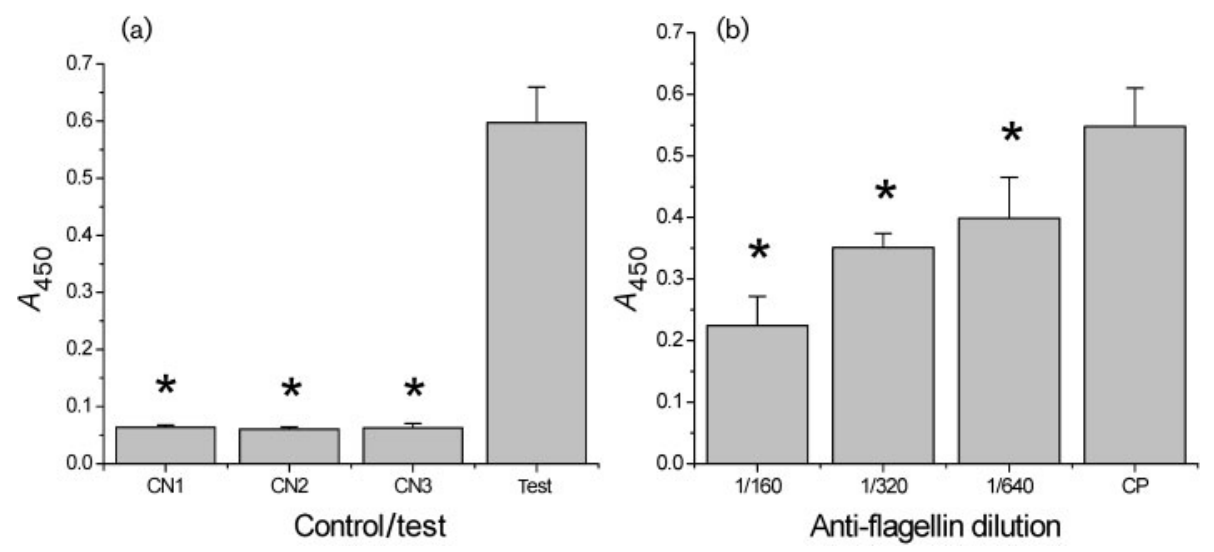

Fig. 6. (a) Ability of flagellin to bind to tracheal mucus as detected by enzyme immunoassay. CN1, Control well coated with mucus, flagellin and normal rabbit serum; CN2, control well coated with mucus and anti-flagellin antibody; CN3, control well with anti-flagellin antibody only. (b) Ability of anti-flagellin antibody to block flagellin attachment to mouse tracheal mucus. The inhibition was antibody concentration dependent. CP, Control well coated with mucus, flagellin and anti-flagellin antibody. 
infection (Thorton et al., 2008). The bacteria are eventually cleared through mucociliary clearance (Bals et al., 1999). This may explain the limited pathogenicity of $S$. maltophilia in a healthy host, despite its ability to adhere to airway epithelial cells, which is equivalent to that of $P$. aeruginosa ( $\mathrm{Wu}$ et al., 2005). As a consequence, S. maltophilia is able to cause disease in those individuals who are severely debilitated or immunosuppressed (Graff \& Burns, 2002; Miyairi et al., 2005). Exactly what causes these bacteria to establish infection in an immunocompromised host needs to be investigated. It is likely that in this situation the aggregated bacteria may form biofilms by binding to mucus. Yang et al. (2008), working with $P$. aeruginosa, demonstrated that biofilms may not necessarily be attached to a surface within the host but may be attached to mucus or suspended in fluid. In another study, evidence was found for $P$. aeruginosa forming aggregates in a matrix in oxygen-depleted mucus from cystic fibrosis patients (Worlitzsch et al., 2002). As biofilm infections are an example of the interplay between the pathogen and the host's genetic makeup, it is likely that S. maltophilia in biofilm mode may act as a pathogen when the host's immune status is lowered. This may be responsible for the change of status from colonization to infection. Although it is interesting to speculate that such a mechanism may be responsible for infection of a compromised host, the exact role of such bacteria (in biofilm mode) needs to be investigated further in an animal model.

The motility of an organism is considered an important mechanism in the pathogenesis of pneumonia (Feldman et al., 1998). However, loss of motility due to decreased expression of flagella has been shown to help $P$. aeruginosa and S. maltophilia to establish in cystic fibrosis airways (Feldman et al., 1998; Matsumoto et al., 1999). As binding to mucus in this study was found to be through the flagella, binding may hinder the movement of the organisms in the respiratory tract and thus indirectly influence the establishment of these organisms. It is likely that biofilm aggregates attached to mucus by flagella may also protect the organism from the host's immune response. In an earlier study, a similar suggestion was made (Hall-Stoodley \& Stoodley, 2009). It has been postulated that bacteria in biofilm mode form a protective mechanism that imparts a beneficial effect to the organism.

In the present study, an attempt was made to delineate the role of flagellin as an adhesin for mouse tracheal mucus. The interaction of flagellin or anti-flagellin antibody with mouse tracheal mucus or bacteria in different experiments suggested the direct involvement of flagella in binding to mucus. This observation was further corroborated using bacteria that had been mechanically deflagellated in a commercial blender. The extent of adhesion of deflagellated bacteria was significantly less than that of their flagellated counterpart. The interaction of flagellin with anti-flagellin antibody before applying to mucus further confirmed its role as an adhesin for mouse tracheal mucus. However, we suggest that the use of genetically defined non-flagellated mutants in future studies will help further in elucidating the exact role of flagella in this process.

In conclusion, our results suggest that the attachment of $S$. maltophilia to mucus may result in either colonization or infection depending on the immune status of the host. Further studies employing animal models will help in understanding the exact mechanism of virulence of this organism, especially in an immunocompromised host.

\section{REFERENCES}

Arora, S. K., Ritchings, B. W., Almira, E. C., Lory, S. \& Ramphal, R. (1998). The Pseudomonas aeruginosa flagellar cap protein, FliD, is responsible for mucin adhesion. Infect Immun 66, 1000-1007.

Baker, P. W. \& Leff, L. G. (2005). Attachment to stainless steel by Mir Space Station bacteria growing under modeled reduced gravity at varying nutrient concentrations. Biofilms 2, 1-7.

Bals, R., Weiner, D. J. \& Wilson, J. M. (1999). The innate immune system in cystic fibrosis lung disease. J Clin Invest 103, 303-307.

Chhibber, S. \& Zgair, A. K. (2009). Involvement of Stenotrophomonas maltophilia flagellin in bacterial adhesion to airway biotic surfaces: an in vitro study. Am J Biomed Sci 1, 188-195.

Cogan, T. A., Jørgensen, F., Lappin-Scott, H. M., Benson, C. E., Woodward, M. J. \& Humphrey, T. J. (2004). Flagella and curli fimbriae are important for the growth of Salmonella enterica serovars in hen eggs. Microbiology 150, 1063-1071.

Di Bonaventura, G., Spedicato, I., D’Antonio, D., Robuffo, I. \& Piccolomini, R. (2004). Biofilm formation by Stenotrophomonas maltophilia: modulation by quinolones, trimethoprim-sulfamethoxazole, and ceftazidime. Antimicrob Agents Chemother 48, 151160.

Feldman, M., Bryan, R., Rajan, S., Scheffler, L., Brunnert, S., Tang, H. \& Prince, A. (1998). Role of flagella in pathogenesis of Pseudomonas aeruginosa pulmonary infection. Infect Immun 66, 43-51.

Girón, J. A. (1995). Expression of flagella and motility by Shigella. Mol Microbiol 18, 63-75.

Graff, G. R. \& Burns, J. L. (2002). Factors affecting the incidence of Stenotrophomonas maltophilia isolation in cystic fibrosis. Chest 121, 1754-1760.

Hall-Stoodley, L. \& Stoodley, P. (2009). Evolving concepts in biofilm infections. Cell Microbiol 11, 1034-1043.

Jucker, B. A., Harms, H. \& Zehnder, A. J. (1996). Adhesion of the positively charged bacterium Stenotrophomonas (Xanthomonas) maltophilia 70401 to glass and Teflon. J Bacteriol 178, 5472-5479.

Katsikogianni, M. \& Missirlis, Y. F. (2004). Concise review of mechanisms of bacterial adhesion to biomaterials and of techniques used in estimating bacteria-material interactions. Eur Cell Mater 8, 37-57.

Matsumoto, T., Tateda, K., Miyazaki, S., Furuya, N., Ohno, A., Ishii, Y., Hirakata, Y. \& Yamaguchi, K. (1999). Effect of antiflagellar human monoclonal antibody on gut-derived Pseudomonas aeruginosa sepsis in mice. Clin Diagn Lab Immunol 6, 537-541.

Miyairi, I., Franklin, J. A., Andreansky, M., Knapp, K. M. \& Hayden, R. T. (2005). Acute necrotizing ulcerative gingivitis and bacteremia caused by Stenotrophomonas maltophilia in an immunocompromised host. Pediatr Infect Dis J 24, 181-183.

Oztuna, F., Ozlü, T., Bülbül, Y., Buruk, K. \& Topbaş, M. (2006). Does cold environment affect Streptococcus pneumoniae adherence to rat buccal epithelium? Respiration 73, 546-551. 
Sajjan, U. S., Corey, M., Karmali, M. A. \& Forstner, J. F. (1992). Binding of Pseudomonas cepacia to normal human intestinal mucin and respiratory mucin from patients with cystic fibrosis. J Clin Invest 89, 648-656.

Thorton, D. J., Rousseau, K. \& McGuckin, M. A. (2008). Structure and function of the polymeric mucins in airways mucus. Annu Rev Physiol 70, 459-486.

Vishwanath, S. \& Ramphal, R. (1984). Adherence of Pseudomonas aeruginosa to human tracheobronchial mucin. Infect Immun 45, 197202.
Worlitzsch, D., Tarran, R., Ulrich, M., Schwab, U., Cekici, A., Meyer, K. C., Birrer, P., Bellon, G., Berger, J. \& other authors (2002). Effects of reduced mucus oxygen concentration in airway Pseudomonas infections of cystic fibrosis patients. J Clin Invest 109, 317-325.

Wu, Q., Lu, Z., Verghese, M. W. \& Randell, S. H. (2005). Airway epithelial cell tolerance to Pseudomonas aeruginosa. Respir Res 6, 26.

Yang, L., Haagensen, J. A., Jelsbak, L., Johansen, H. K., Sternberg, C., Høiby, N. \& Molin, S. (2008). In situ growth rates and biofilm development of Pseudomonas aeruginosa populations in chronic lung infections. J Bacteriol 190, 2767-2776. 